\title{
A Very High-Speed Digital Number Sieve
}

\author{
By D. G. Cantor, G. Estrin, A. S. Fraenkel, and R. Turn
}

1. Introduction. The general sieve problem may be stated as follows [3]. Let $m_{1}, m_{2}, \cdots, m_{s}$ be $s$ positive integers, relatively prime in pairs. Consider the congruences

$$
x \equiv a_{i j}\left(\bmod m_{i}\right), \quad i=1,2, \cdots, s ; j=1,2, \cdots, t_{i}<m_{i} .
$$

For fixed $i$, the $a_{i j}$ are distinct non-negative integers less than $m_{i}$. The problem is to find all integers $N$ between given limits, say

$$
A \leqq N<B
$$

such that $N$ is a solution to $s$ of the congruences. (It is, of course, clear that no $N$ can be a solution to more than $s$ of the congruences (1).)

Examples: On the one extreme there is the Sieve of Eratosthenes for finding all primes $p$ in the range $A=B^{1 / 2} \leqq p<B$, where $t_{i}=m_{i}-1$ for all $i$. (Here $m_{i}$ are all the primes $<B^{1 / 2}$.) On the other extreme there is the Chinese remainder type of problem, where $t_{i}=1$ for all $i$, and there is only one solution among $\prod_{i=1}^{s} m_{i}$ numbers.

In between these two extremes, there is the important quadratic sieve, where roughly $t_{i}=m_{i} / 2$ for all $i$. It is used in problems involving quadratic residues, Diophantine equations of second degree and other quadratic type problems.

About thirty years ago, Lehmer [1], [2] constructed a novel special-purpose device for sifting. It used the first 30 primes as moduli. Its processing rate was

$$
3 \times 10^{5} \text { numbers } / \mathrm{min} \text {. }
$$

General-purpose computers are not very well suited to sifting, and the earlier models could not compete with Lehmer's machine. However, the speed of the more recent machines makes up for their lack of orientation towards the sieve problem insofar as surpassing the performance of Lehmer's machine is concerned. Thus, the rate for a quadratic sieve using the first 30 primes on the IBM 7090 is approximately

$$
10^{7} \text { numbers/min. }
$$

The present paper describes a special-purpose device, where rates in excess of $10^{10}$ numbers/min.

can be achieved for quadratic sieves. The device consists of basic digital building blocks from which a suitable sieve is assembled for each problem. Thus, by an appropriate rearrangement of the building blocks, problems with different moduli can be run. It is also shown that if the device contains a certain minimum amount of hardware and is attached to a general-purpose computer, then problems can be run where, roughly speaking, the number of moduli is not limited any more by the

Received June 23, 1961. The preparation of this paper was sponsored, in part, by the Office of Naval Research and the Atomic Energy Commission. 
amount of hardware of the special-purpose device, but only by the size of the memory of the general-purpose computer, and the rate is still of the order of $10^{10}$ numbers/min. We use a so-called "Fixed Plus Variable Structure Computer" organization for realizing the combination between the special- and general-purpose computers [6]. This also enables one to use the digital building blocks of the sieve for building other special-purpose devices which one might want to associate with the general-purpose computer.

2. Binary Set-Up of the Sieve. For solving the system (1) on a digital computer, we consider a matrix $M$ of size $s \times(B-A)$ with entries $c_{i k}(i=1,2, \cdots, s ; k=A$, $A+1, \cdots, B-1)$, defined by

$$
c_{i k}=\left\{\begin{array}{l}
1 \text { if } k \equiv a_{i j} \quad\left(\bmod m_{i}\right) \\
0 \text { otherwise }
\end{array} \quad\left(j=1,2, \cdots, t_{i}\right) .\right.
$$

Then every column, all of whose entries are 1, corresponds to a solution, and conversely.

Example: Find the primes $p$ such that

$$
6 \leqq p<36
$$

The relevant congruences are $x \equiv 1(\bmod 2), x \equiv 1,2(\bmod 3), x \equiv 1,2,3,4$ $(\bmod 5)$. The matrix $M$ is given by

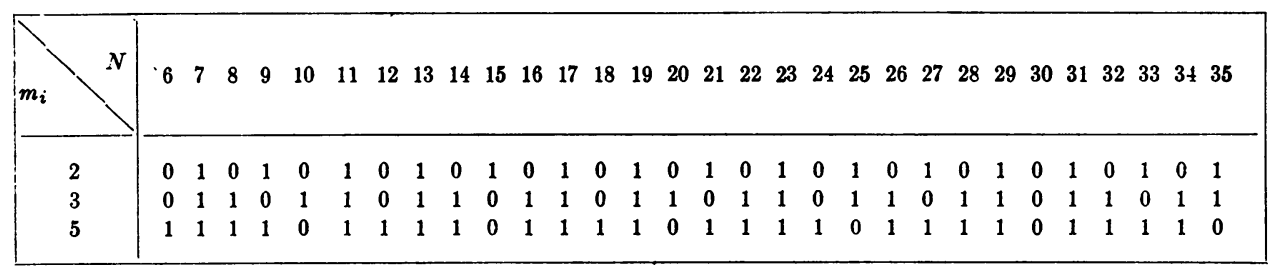

The columns all of whose entries are 1 correspond to the primes in the range (3).

The rows of $M$ are periodic with period $m_{i}$. Thus, the first ordered $m_{i}$ bits of the $i$ th row determine the rest of this row completely, and we call them the periodic pattern $e_{i}$ of $m_{i}$.

3. Method of Solution on the Special-Purpose Computer. We now give an informal introduction to the principle of operation of the special-purpose device. It will be observed that the method is based on ideas used in earlier work [1], [2], [3] in this field.

A first approach to the mechanization of a special-purpose sieve would be to build a matrix precisely in the form displayed for the example above with observation posts in every column, detecting coincidences of non-zero bits. Problems which can be solved by such a procedure are limited to those which can fit into the maximum size matrix which can be assembled, i.e., computationally trivial problems.

We order the moduli so that

$$
m_{1}<m_{2}<\cdots<m_{s} .
$$


Since solutions exist only corresponding to columns with non-zero bits, we may eliminate the $m_{s}$-row and many of the components required to detect coincidences, by establishing coincidence gates only in those columns where the $m_{s}$-row has non-zero entries.

Large problems may be handled by constructing only $m_{s}$ columns of the matrix and testing for solutions in these columns in parallel. Entering the next batch of $m_{s}$ numbers turns out to be equivalent to performing prearranged circular shifts in the $s-1$ rows. This procedure would require a matrix of size $(s-1) \times m_{s}$ with coincidence gates established in the columns as prescribed above. It is possible to use such a matrix to define potential solutions even when it is only feasible to mechanize $l<s-1$ rows of the matrix, and then have a general-purpose computer complete the test for solution.

The range of problems which may be handled is increased when it is recognized that the periodic pattern $e_{i}$ associated with the $i$ th row completely determines the rest of the row. In the following we give an algorithm which defines a procedure requiring only $m_{i}$ elements in the $i$ th row, giving up only the regularity of the coincident gate connections. The special-purpose computer consists of basic digital building blocks or modules which are assembled into a matrix consisting of $s-1$ shifting registers, the $i$ th of length $m_{i}$, and initially containing the periodic pattern $e_{i}(i=1,2, \cdots, s-1)$. Observation posts are placed at certain positions in the matrix which sift out the solutions to (1) among the first $m_{s}$ numbers. ${ }^{*}$ Next, a circular shift is performed in each register, which is equivalent to bringing in the next $m_{s}$ numbers to be sifted. This is followed by the observation posts sifting out the solutions among this new batch of numbers. This process of sifting followed by shifting is continued until all the numbers are processed.

4. The Algorithm. We divide the numbers $N$ in the range (2) into sets $S_{n}$ defined by $\dagger$

(5) $S_{n}=\left\{N: N<B, N=A+n m_{8}+k ; 0 \leqq k<m_{s}\right\}, \quad n=0,1, \cdots,\left[\frac{B-A-1}{m_{8}}\right]$.

Thus, each set (except possibly the last) contains $m_{s}$ numbers.

Let

$$
m_{s}=q_{i} m_{i}+r_{i}, \quad 0<r_{i}<m_{i} \quad(i=1,2, \cdots, s-1) .
$$

With each set $S_{n}$ we associate a matrix $M_{n}$ of size $(s-1) \times m_{s}$ with entries $c_{i j}(n)\left(i=1,2, \cdots, s-1 ; j=0,1, \cdots, m_{s}-1\right)$, defined recursively by

$$
c_{i j}(0)=\left\{\begin{array}{l}
1 \text { if } 0 \leqq j<m_{i} \text { and } A+j \equiv a_{i j}, \cdots, a_{i}, t_{i}\left(\bmod m_{i}\right) \\
0 \text { otherwise. }
\end{array}\right.
$$

$$
c_{i j}(n)=\left\{\begin{array}{l}
c_{i, j+r_{i}}(n-1) \quad \text { if } 0 \leqq j+r_{i}<m_{i} \\
c_{i, j+r_{i}-m_{i}}(n-1) \text { if } 0 \leqq j<m_{i} \text { and } j+r_{i} \geqq m_{i} \\
0 \text { if } m_{i} \leqq j<m_{s} .
\end{array}\right.
$$

* The positioning of the observation posts is determined by $m_{6}$ and its residues in such a way that a register of length $m_{s}$ is not required.

$\dagger[x]$ stands for the largest integer $\leqq x$. 
Equation (8) can be written in the form

$$
c_{i j}(n)=\left\{\begin{array}{l}
c_{i d}(n-1) \quad \text { where } m_{i}>d \equiv j+r_{i}\left(\bmod m_{i}\right), \text { if } 0 \leqq j<m_{i} \\
0 \text { if } m_{i} \leqq j<m_{s} .
\end{array}\right.
$$

Hence (7) and (8) are equivalent to

(9) $\quad c_{i j}(n)= \begin{cases}1 & \text { if } 0 \leqq j<m_{i} \text { and } A+j \equiv a_{i, v_{i}}-n r_{i}\left(\bmod m_{i}\right) \\ 0 & \text { otherwise } \\ & \left(v_{i}=1,2, \cdots, t_{i}\right) .\end{cases}$

If $N \in S_{n}$ is a solution to the system (1), then by (5),

$$
A+k \equiv a_{s, v_{s}}\left(\bmod m_{s}\right) \quad\left(0 \leqq k<m_{s} ; v_{s}=1,2, \cdots, t_{s}\right)
$$

for all $n$.

By (5) and (6) we have also

(11) $A+k \equiv a_{i, v_{i}}-n r_{i}\left(\bmod m_{i}\right) \quad\left(v_{i}=1,2, \cdots, t_{i} ; i=1,2, \cdots, s-1\right)$.

Hence, if we let

$$
k=w_{i} m_{i}+u_{i}, \quad 0 \leqq u_{i}<m_{i} \quad(i=1,2, \cdots, s-1),
$$

then by (11),

$$
A+u_{i} \equiv a_{i, v_{i}}-n r_{i}\left(\bmod m_{i}\right) \quad\left(v_{i}=1,2, \cdots, t_{i} ; i=1,2, \cdots, s-1\right),
$$

so that

$$
c_{i, u_{i}}(n)=1
$$

for $i=1, \cdots, s-1$ by $(9)$.

Also the converse holds. That is to say, if (13) holds for $i=1, \cdots, s-1$ (where $u_{i}$ is given by (12) and $k$ by $(10)$ ), then

$$
N=A+n m_{s}+k
$$

is a solution to the system (1). This is the basis of the algorithm. We list the $t_{s}$ solutions $k_{1}, \cdots, k_{t_{s}}$ of $(10)$, and for each of them its corresponding $s-1$ values $u_{i}$. Then the numbers $N=A+n m_{s}+k_{v_{s}}\left(v_{s}=1, \cdots, t_{s}\right)$ for which (13) holds for $i=1, \cdots, s-1$, are solutions to (1), and these are all the solutions.

Example: Find all solutions in the range

$$
-15 \leqq N<25
$$

to the system of congruences

$$
\begin{array}{ll}
x \equiv 1 & (\bmod 2) \\
x \equiv 1,2 & (\bmod 3) \\
x \equiv 2,3,4 & (\bmod 5) \\
x \equiv 0,1,2,5 & (\bmod 7) \\
x \equiv 0,1,8,9 & (\bmod 11) .
\end{array}
$$

Reference is made to Table $I$. The matrix $M_{0}$ is constructed according to ( 7 ) (omitting all strings of zeros). Equation (8) implies that the $i$ th row of matrix $M_{n}$ is obtained from the $i$ th row of $M_{n-1}$ by means of circularly left shifting the 
TABLE I

The Matrices for the Problem

\begin{tabular}{c|ccccccccccc|}
\hline & $N$ \\
& $\Upsilon_{j}$ \\
\hline 1 & 0 & 1 & 2 & 3 & 4 & 5 & 6 & 7 & 8 & 9 & 10 \\
\hline 2 & 1 & 0 & & & & & & & & \\
3 & 0 & 0 & 1 & 1 & 1 & & & & $M_{0}$ & & \\
4 & 0 & 1 & 1 & 1 & 0 & 0 & 1 & & & & \\
\hline
\end{tabular}

\begin{tabular}{|c|ccccccccccc|}
\hline$i Y_{j}$ & -4 & -3 & -2 & -1 & 0 & 1 & 2 & 3 & 4 & 5 & 6 \\
& 0 & 1 & 2 & 3 & 4 & 5 & 6 & 7 & 8 & 9 & 10 \\
\hline 1 & 0 & 1 & & & & & & & & & \\
2 & 1 & 0 & 1 & & & & & & $M_{1}$ & & \\
3 & 0 & 1 & 1 & 1 & 0 & & & & & & \\
4 & 0 & 0 & 1 & 0 & 1 & 1 & 1 & & & & \\
\hline
\end{tabular}

\begin{tabular}{|c|ccccccccccc|}
\hline$i N$ & 7 & 8 & 9 & 10 & 11 & 12 & 13 & 14 & 15 & 16 & 17 \\
$i$ & 0 & 1 & 2 & 3 & 4 & 5 & 6 & 7 & 8 & 9 & 10 \\
\hline 1 & 1 & 0 & & & & & & & & \\
2 & 1 & 1 & 0 & & & & & & $M_{2}$ & & \\
3 & 1 & 1 & 1 & 0 & 0 & & & & & & \\
4 & 1 & 1 & 1 & 0 & 0 & 1 & 0 & & & & \\
\hline
\end{tabular}

\begin{tabular}{|c|ccccccc|}
\hline$i$ & 18 & 19 & 20 & 21 & 22 & 23 & 24 \\
$i$ & 0 & 1 & 2 & 3 & 4 & 5 & 6 \\
\hline 1 & 0 & 1 & & & & & \\
2 & 0 & 1 & 1 & & & $M_{3}$ & \\
3 & 1 & 1 & 0 & 0 & 1 & & 0 \\
4 & 0 & 1 & 0 & 1 & 1 & 1 & 0 \\
\hline
\end{tabular}

first $m_{i}$ bits by $r_{i}$ positions (or by circularly right shifting them by $m_{i}-r_{i}$ positions). In the present case, $r_{1}=1, r_{2}=2, r_{3}=1, r_{4}=4$, so that in passing from one matrix to the next, the first row is shifted left circularly by 1 position, the second by 2 , the third by 1 and the fourth by 4 positions. This is the way $M_{1}$, $M_{2}$ and $M_{3}$ are obtained.

The values $k_{1}, k_{2}, k_{3}, k_{4}$ of Table II are computed by (10), and the corresponding values of $u_{i}$ by (12). Table II defines a pattern of observation stations which sift out the solutions in each matrix; the entry $u_{i}$ represents the column coordinate corresponding to the row coordinate $i$, at which an observation station exists. In $M_{0}$, the observation pattern $u_{i}=0,2,2,2$ indicates a solution, since all matrix positions corresponding to that pattern are filled by 1's. They appear in bold type in Table I. The corresponding value of $k$ is $k_{4}=2$. The solution is, therefore, $N=-15+0 \times 11+2=-13$ by (14). The only two other solutions 
TABLE II

Observation Posts for the Problem

\begin{tabular}{|c|c|c|c|c|c|}
\hline \multirow{2}{*}{$i$} & \multirow{2}{*}{$m_{i}$} & \multicolumn{4}{|c|}{$U_{i}$} \\
\hline & & $k_{1}=4$ & $k_{2}=5$ & $k_{3}=1$ & $k_{4}=2$ \\
\hline 1 & 2 & 0 & 1 & 1 & 0 \\
\hline 2 & 3 & 1 & 2 & 1 & 2 \\
\hline 3 & 5 & 4 & 0 & 1 & 2 \\
\hline 4 & 7 & 4 & 5 & 1 & 2 \\
\hline
\end{tabular}

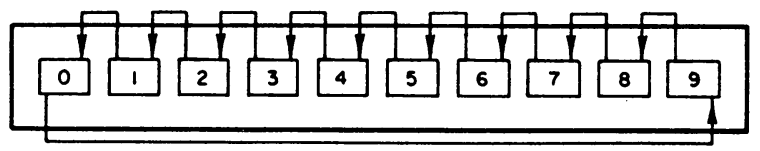

Fig. 1.-Conventional Shift Register.

are found in $M_{3}$, for $k_{3}=1$ and $k_{2}=5$, also indicated by bold type in Table I. They are $N=-15+3 \times 11+1=19$ and $N=-15+3 \times 11+5=23$.

5. The Special-Purpose Computer. The special-purpose device should be flexible enough to allow usage of different moduli. Therefore the basic building blocks of the device are modules consisting of memory elements and gates which will be assembled into the appropriate-size shifting registers and sets of and-gates for each problem. The and-gates are the realization of appropriate combinations of the observation posts, and test for coincidence of 1's.

The example of the previous section suggests the construction of the device. Its central part consists of shift-registers $R_{1}, \cdots, R_{s-1}$, the $i$ th of length $m_{i}$, which will store the matrices $M_{n}$ (without the trailing strings of 0 's). Register $R_{i}$ will shift circularly left by $r_{i}$ positions. It is important to note, however, that this can be effected in one shift time, rather than in $r_{i}$ shift times, and further, that the wiring can be so arranged that any transmitting memory element is adjacent to its receiver. In order to do this, we rename the memory elements in $R_{i}$ so that element number 0 is at the left, followed by element number $r_{i}$, followed by number $2 r_{i}\left(\bmod m_{i}\right)$, by $3 r_{i}\left(\bmod m_{i}\right)$, etc. Then each transmitting element is adjacent to its receiver, and every element will appear exactly once. For $\ddagger\left(m_{i}, m_{s}\right)=1$, so that also $\left(m_{i}, r_{i}\right)=1(i=1, \cdots, s-1)$. Hence $r_{i}$ generates the additive cyclic group of non-negative integers $\left(\bmod m_{i}\right)$ and every element appears exactly once in the register.

Example: Suppose that for a certain sieve problem $m_{s}=23$, and $m_{i}=10$ for some $i<s$. Then $R_{i}$ has to shift circularly left by $r_{i}=3$ positions. On a conventional shift-register, three shifts of the type indicated in Figure 1 would have to be performed. The same result can be obtained in one shift time by the specially wired-up register of Figure 2. However, the long wiring has an undesirable effect on the speed of the system. Renaming the memory elements as in Figure 3, the three shifts can be done in one shift time with conventional wiring.

$\ddagger(a, b)$ stands for the greatest common divisor of $a$ and $b$. 


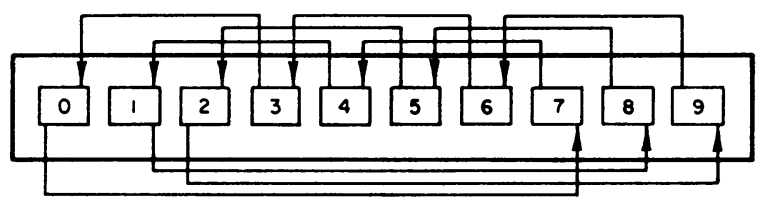

Fig. 2.-Specially Wired-Up Register for Performing a Shift of Three Positions in One Shift Time.

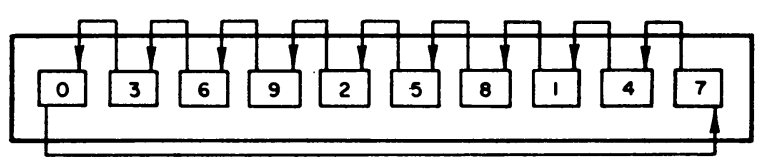

FIG. 3.-Final Form of the Register.

The second part of the special-purpose device consists of $t_{s}$ sets of and-gates, one set for each value of $k$ which is a solution to (10), "anding" together positions $c_{i j}$ in the registers, as defined in the previous section. Also a counting register $R$ with capacity $>(B-A) / m_{8}$ and a shift control are required. (The shift control does not normally have to be reconstructed for every problem.) The register $R$ contains the value $n$ of the matrix $M_{n}$ currently being sifted.

The sifting process consists of the following steps.

1. Clear $R$.

2. Load $R_{1}, \cdots, R_{s-1}$ with $M_{0}$, whose entries are defined by (7).

3. Record $n$ and record the values $k$ for which coincidence is obtained, i.e., the values $k$ associated with the sets of and-gates which are excited, if any. The corresponding solutions are given by (14).

4. Advance $R$ by unity and perform a circular shift in each shift-register according to the above scheme. This effectively reloads the registers with the next batch of $m_{s}$ numbers to be sifted.

5. Terminate process if $n>(B-A) / m_{s}$. Otherwise go back to 3 .

It is thus seen that in this process $m_{s}$ numbers are processed per shift time.

6. The Sieve in a Fixed Plus Variable Structure Computer. It was remarked by Lehmer that special-purpose equipment attached to the arithmetic unit of a fast computer can speed up computation of permutation problems [4], and of other problems [5]. More generally, we consider a so-called "Fixed Plus Variable Structure Computer" (to be designated by $(F+V)$ computer), which consists of a conventional digital computer (the fixed part to be denoted by $F$ ), and a set of modules (the variable part to be denoted by $V$ ). Many problems contain a part which can be solved on a special-purpose computer in a much more efficient way than on a general-purpose computer. For such a problem, the modules are assembled into a suitable special-purpose device which handles this part. The rest of the problem is handled by $F$. A supervisory control coordinates the operation of the two computers. However, the special-purpose configuration is not retained permanently, but may be reorganized into other configurations for other problems. For a more detailed description of the concept of the $(F+V)$ computer, the reader is referred to the literature [6].

The special-purpose device described above has a limited amount of hardware. 


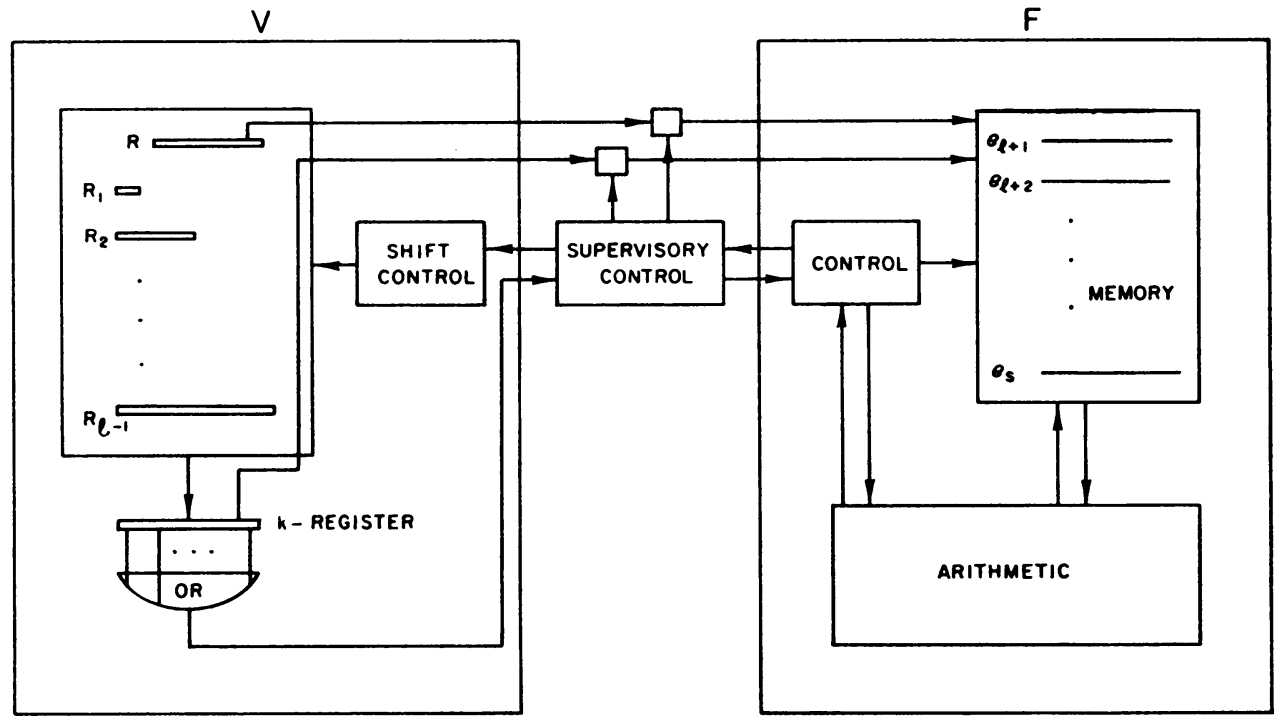

FIg. 4. $-(F+V)$ Computer Organization for the Sieve Problem.

For certain problems it may be desirable to use more moduli than can be mechanized with the available hardware. In order to handle such problems, we imbed the special-purpose device in an $(F+V)$ computer. This allows, as will be shown subsequently, handling problems in which the number of moduli is limited only by the number of periodic patterns $e_{i}$ of $m_{i}$ that can be stored in the memory of $F$, provided that the hardware of $V$ is sufficient to mechanize the first $l$ of the $s$ moduli. The parameter $l$ depends on the relative number of 1 's and 0 's in the periodic patterns of the sieve, and on the relative speeds of $F$ and $V$. The use of $F+V$ also allows using the modules for building special-purpose devices other than the sieve, and attaching them to $F$.

Figure 4 shows the organization of the $(F+V)$ computer for the sieve problem by means of a block diagram. The $V$-part, which acts as the special-purpose device, mechanizes the first $l$ moduli. The $k$-register records the values of $k$ (the solutions of (10)) corresponding to coincidence. The periodic patterns $e_{l+1}, \cdots, e_{s}$ of $m_{l+1}, \cdots, m_{s}$ are stored in the memory of $F$. Numbers will be sifted in $V$, and when coincidence occurs, the contents of $R$ and of the $k$-register are transferred to $F$, where so-called solution candidates $N$ of the form (14) are formed, one for each value of $k$. Then divisions of the form

$$
N=\sigma_{i} m_{i}+\rho_{i}, \quad 0 \leqq \rho_{i}<m_{i} \quad(i=l+1, \cdots, s)
$$

are performed in $F$. The residue $\rho_{i}$ determines uniquely the position of the bit of the periodic pattern $e_{i}$ of $m_{i}$ corresponding to $N$. The number $N$ is a solution if and only if these bits are 1 for $i=l+1, \cdots, s$. Thus, the $(F+V)$ computer is so organized that $V$ will do the high-speed sifting, and $F$ will do divisions. $l$ will be chosen so that the average time per coincidence in $V$ is at least as large as the average division time per coincidence in $F$. Then $V$ would normally do its divisions, until such time when coincidence is obtained in $V$. At such time, $V$ is interrupted 
and the transfers from $V$ to the memory of $F$ occur, the latter acting as a buffer, capable of storing "bursts" of solution candidates which $V$ might produce occasionally. After the transfers, both parts are again decoupled and assume their respective tasks. The program for $V$ is outlined in the following six steps.

1. Clear $R$.

2. Load $R_{1}, \cdots, R_{l-1}$ with $M_{0}$.

3. Check for coincidence. If none is obtained, go to 5 . Otherwise continue with 4 .

4. Interrupt $F$ and $V$. Transfer the contents of $R$ and of the $k$-registers to the memory of $F$.

5. Advance $R$ by unity and perform a circular left shift of $r_{i}$ places in $R_{i}(i=$ $1, \cdots, l-1)$.

6. Terminate process if $n>(B-A) / m_{l}$. Otherwise go back to 3 .

The program for $F$ is simply to produce solution candidates $N$ of the form (14), and to perform divisions of the type (15) for each of them until the first 0-bit is encountered. If none is encountered, $N$ is recorded as a solution.

7. Speed and Hardware. The speed and hardware requirements will now be discussed in terms of an example, for which we choose a quadratic sieve problem where the moduli are the first $s$ primes. The first column of Table III contains values of the independent variable $l$, the number of moduli mechanized in the special-purpose device. The table displays the speed and hardware requirements for such a sieve as a function of $l$. The second column contains the $l$ th prime. Let $t$ be the total time required for performing the coincidence test and the subsequent circular shifts in the registers. Using the register organization described in Section 5 , the circular shifting amounts to a left shift of one position in each register. We assume transistorized circuitry, for which

$$
t=0.2 \mu \mathrm{sec}
$$

is chosen (speeds approximating those of the IBM 7090). Thus, $m_{l}$ numbers are processed in this time if no coincidence occurs. (If the registers are of the doublerank type, both ranks will be equipped with sets of and-gates, and $t=0.2 \mu \mathrm{sec}$ is the time for a coincidence check and for transferring one rank into the other. Thus also in this case $m_{l}$ numbers are processed in $0.2 \mu$ sec.)

We consider first the case $s=l$, that is, we use only a special-purpose computer without a conventional general-purpose computer. Assuming the solutions to be sparse, so that we may neglect the time of recording them, the rate of the sieve is

$$
v=\frac{6 \times 10^{7} \times m_{l}}{t}=3 \times 10^{8} \times m_{l} \text { numbers } / \mathrm{min} \text {. }
$$

These values are displayed in the third column.

Since the sieve is quadratic, the probability of any randomly selected bit in the periodic pattern $e_{i}$ to be 1 is about 0.5 . Hence, on the average, one coincidence is obtained per $2^{l}$ numbers sifted, or every

$$
\tau=\frac{6 \times 10^{7} \times 2^{l}}{v}=\frac{2^{l}}{5 m_{l}} \mu \text { sec. }
$$

These values appear in the fourth column. 
If $s>l$, divisions have to be performed in $F$. The probability that exactly $i$ divisions suffice to decide whether any solution candidate $N$ has to be rejected or accepted is $(1 / 2)^{i}(1 \leqq i \leqq s-l)$. Hence the expectation of the number of divisions for each $N$ is given by

$$
\nu=\sum_{i=1}^{s-l} i / 2^{i}=2-(s-l+2) / 2^{s-l} .
$$

Thus the average number of divisions for each solution candidate approaches 2 asymptotically from below. Assuming the IBM 7090 as the fixed machine $F$, this division subroutine takes about $200 \mu \mathrm{sec}$ for two divisions. Also, preliminary studies of the mode of transfer from $V$ to the 7090 indicate that the transfer of the contents of $R$ and of the $k$-register requires no more than $7 \mu$ sec. (See appendix.) That is, this is the maximum time during which $V$ is idle. $F$ is interrupted only insofar as it requires memory access during this time. Actually, $V$ could already resume its operation after the transfer of $n$ from the $R$-register. It would have to wait additional time only if a new solution candidate is formed before the current contents of the $k$-register has been stored away, which is a rare event. However, in our computation of the overall speed of the sieve we assumed that $V$ is interrupted for $7 \mu$ sec. during each transfer.

Thus, the average overall rate of the sieve is given by

$$
w=\frac{v}{1+7 / \tau} \text { numbers } / \mathrm{min} \text {. }
$$

for $\tau \geqq 200 \mu$ sec. If $\tau<200 \mu$ sec, $V$ will have to wait for $F$, and the average overall rate for this case is

$$
w=\frac{\tau}{200} \frac{v}{1+7 / \tau} \text { numbers } / \mathrm{min} .
$$

Thus, the operation of the sieve becomes rapidly more and more inefficient as $\tau$ decreases below the critical value of $200 \mu$ sec. The values of $w$ appear in the fifth column of Table III. The last column displays the required number $h=\sum_{i=1}^{l-1} m_{i}$ of memory elements for the special-purpose device. (This number has to be doubled if the registers are of the double-rank type.)

The lower bound for $l$ in Table III was chosen to be 9 because for $l=8$ the rate would already be less than can be achieved with conventional present-day computers. The upper bound was chosen by setting arbitrarily a hardware constraint of 1500 memory elements.

The lowest value of $\tau$ for which $\tau \geqq 200 \mu \mathrm{sec}$ is $\tau=247.3 \mu \mathrm{sec}$. Thus $V$ should contain at least 15 registers consisting of 328 memory elements. Figure 5 displays the overall rate as a function of required memory elements. Two simple conclusions can be drawn from the monotonicity of $w$ as displayed in Figure 5. First, $l$ should be chosen as large as possible. That is to say, as much hardware as available should be thrown in to build the sieve; even so the cooperation between $F$ and $V$ becomes less efficient as $l$ increases beyond the critical value of 16 , in the sense that $F$ becomes more idle. Secondly, the use of slower memory elements is indicated if a larger number of them is available, hence the possibility of using magnetic core registers. 
TABLE III

\begin{tabular}{|c|c|c|c|c|c|}
\hline \multicolumn{6}{|c|}{ Speed and Hardware as a Function of $l$ For a Quadratic Sieve } \\
\hline $\begin{array}{c}\text { l-No. of Moduli } \\
\text { Implemented in } \\
\text { Special-Purpose } \\
\text { Device }\end{array}$ & $\begin{array}{l}m_{l} \text {-the } \\
l \text { th } \\
\text { Modulus }\end{array}$ & $\begin{array}{l}v-\text { Numbers/ } \\
\text { Min. Rate of } \\
\text { Sieve if } s=l\end{array}$ & $\begin{array}{l}\tau \text { Average Time } \\
\text { Per Coincidence }\end{array}$ & $\begin{array}{l}w-\text { Numbers/ } \\
\text { Min. Rate of } \\
\text { Sieve if } s>l\end{array}$ & $\begin{array}{c}h-\text { Number of } \\
\text { Memory Ele- } \\
\text { ments in Special- } \\
\text { Purpose Device }\end{array}$ \\
\hline 9 & 23 & $6.9 \times 10^{9}$ & $4.5 \mu \mathrm{sec}$ & $5.70 \times 10^{7}$ & 77 \\
\hline 10 & 29 & $8.7 \times 10^{9}$ & $7.1 \mu \mathrm{sec}$ & $1.56 \times 10^{8}$ & 100 \\
\hline 11 & 31 & $9.3 \times 10^{9}$ & $13.2 \mu \mathrm{sec}$ & $4.03 \times 10^{8}$ & 129 \\
\hline 12 & 37 & $1.11 \times 10^{10}$ & $22.1 \mu \mathrm{sec}$ & $9.28 \times 10^{8}$ & 160 \\
\hline 13 & 41 & $1.23 \times 10^{10}$ & $39.9 \mu \mathrm{sec}$ & $2.09 \times 10^{9}$ & 197 \\
\hline 14 & 43 & $1.29 \times 10^{10}$ & $76.2 \mu \mathrm{sec}$ & $4.49 \times 10^{9}$ & 238 \\
\hline 15 & 47 & $1.41 \times 10^{10}$ & $139.4 \mu \mathrm{sec}$ & $9.34 \times 10^{9}$ & 281 \\
\hline 16 & 53 & $1.59 \times 10^{10}$ & $247.3 \mu \mathrm{sec}$ & $1.54 \times 10^{10}$ & 328 \\
\hline 17 & 59 & $1.77 \times 10^{10}$ & $444.3 \mu \mathrm{sec}$ & $1.73 \times 10^{10}$ & 381 \\
\hline 18 & 61 & $1.83 \times 10^{10}$ & $859 \mu \mathrm{sec}$ & $1.81 \times 10^{10}$ & 440 \\
\hline 19 & 67 & $2.01 \times 10^{10}$ & $1.6 \mathrm{~m} \mathrm{sec}$ & $2.01 \times 10^{10}$ & 501 \\
\hline 20 & 71 & $2.13 \times 10^{10}$ & $2.9 \mathrm{~m} \mathrm{sec}$ & $2.13 \times 10^{10}$ & 568 \\
\hline 21 & 73 & $2.19 \times 10^{10}$ & $5.7 \mathrm{~m} \mathrm{sec}$ & $2.19 \times 10^{10}$ & 639 \\
\hline 22 & 79 & $2.37 \times 10^{10}$ & $10.6 \mathrm{~m} \mathrm{sec}$ & $2.37 \times 10^{10}$ & 712 \\
\hline 23 & 83 & $2.49 \times 10^{10}$ & $20.2 \mathrm{~m} \mathrm{sec}$ & $2.49 \times 10^{10}$ & 791 \\
\hline 24 & 89 & $2.67 \times 10^{10}$ & $37.7 \mathrm{~m} \mathrm{sec}$ & $2.67 \times 10^{10}$ & 874 \\
\hline 25 & 97 & $2.91 \times 10^{10}$ & $69.2 \mathrm{~m} \mathrm{sec}$ & $2.91 \times 10^{10}$ & 963 \\
\hline 26 & 101 & $3.03 \times 10^{10}$ & $132.9 \mathrm{~m} \mathrm{sec}$ & $3.03 \times 10^{10}$ & 1060 \\
\hline 27 & 103 & $3.09 \times 10^{10}$ & $260.6 \mathrm{~m} \mathrm{sec}$ & $3.09 \times 10^{10}$ & 1161 \\
\hline 28 & 107 & $3.21 \times 10^{10}$ & $501.7 \mathrm{~m} \mathrm{sec}$ & $3.21 \times 10^{10}$ & 1264 \\
\hline 29 & 109 & $3.27 \times 10^{10}$ & $985.1 \mathrm{~m} \mathrm{sec}$ & $3.27 \times 10^{10}$ & 1371 \\
\hline 30 & 113 & $3.39 \times 10^{10}$ & $1900 \mathrm{~m} \mathrm{sec}$ & $3.39 \times 10^{10}$ & 1480 \\
\hline
\end{tabular}

By (16), the average number of divisions per solution candidate in $F$ is less than 2 , whatever the number of periodic patterns that are stored in $F$. Therefore, the rate $w$ of a quadratic sieve is practically independent of $s$, and the number of the moduli is limited only by the number of periods that can be stored in $F$. A similar remark applies also for sieves that are "less than quadratic," i.e., where the number of 1 's in $e_{i}$ is $<m_{i} / 2$. For these types of sieves there are even less divisions to be performed, and a higher overall rate is obtained. For sieves that are "more than quadratic," and in particular for those which approach the type of sieve of Eratosthenes, more than two divisions are required on the average, and a higher critical value of $l$ is obtained.

Variations of the above described method which result in even higher speeds (and therefore involve higher critical values of $l$ ) are clearly possible. For example, two moduli may be combined in $V$, say $m_{l}$ and $m_{l-1}$, by initially solving the two congruences involving $m_{l}$ and $m_{l-1}$ manually or on $F$. Then $m_{l} m_{l-1}$ numbers can be processed per shift time, for which $t_{l} t_{l-1}$ sets of and-gates are required. Registers $m_{l}$ and $m_{l-1}$ do not have to be built of course. As another example, we might mechanize the moduli $m_{1}, m_{2}, \cdots, m_{l-1}, m_{s}$ in $V$, rather than $m_{1}, m_{2}, \cdots, m_{l-1}, m_{l}$, so that $m_{s}$ numbers instead of only $m_{l}$ are processed per shift time. 


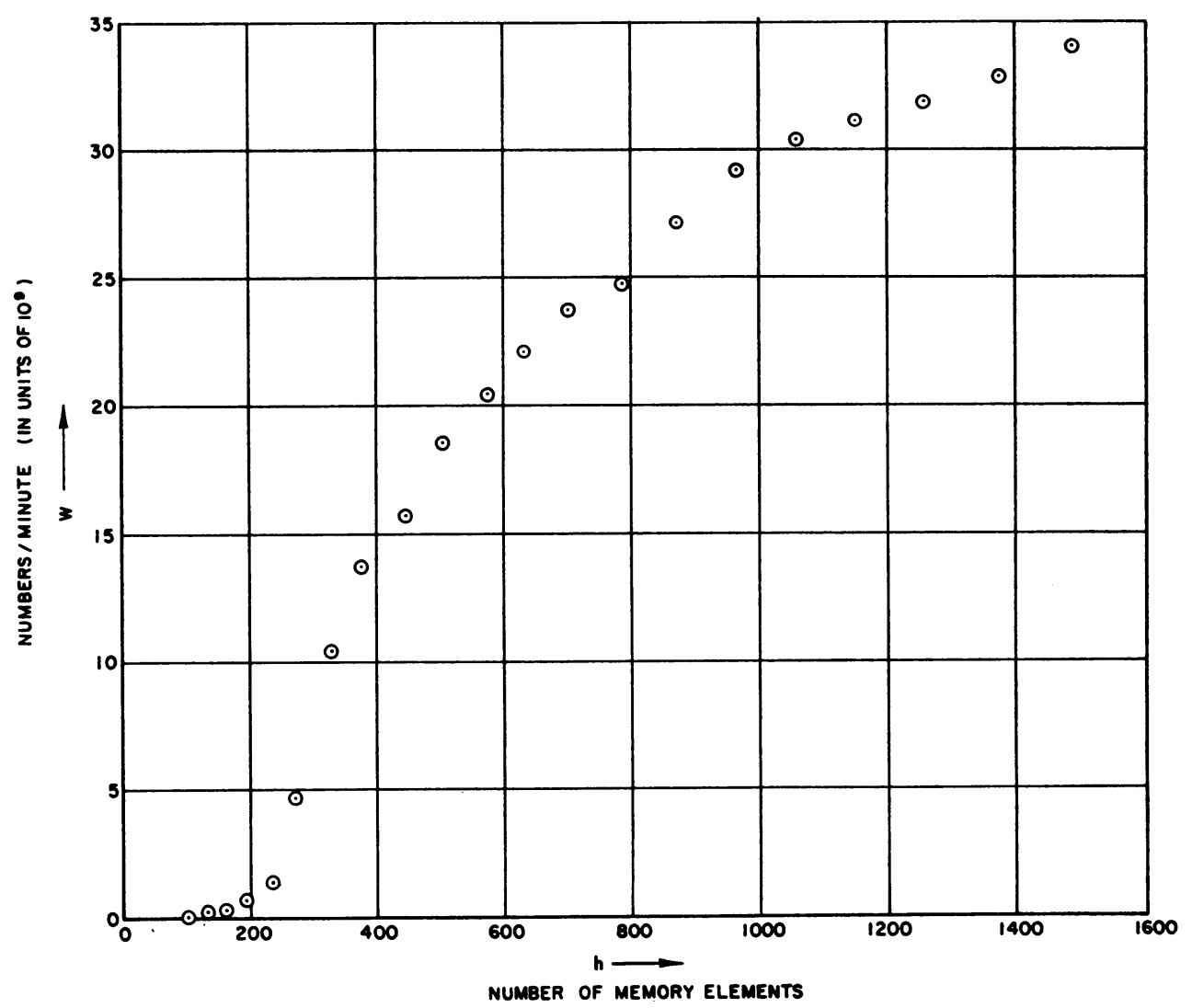

Fig. 5.-Rate of Sieve as a Function of the Number of Memory Elements.

Similarly, two moduli may be combined in $F$. For example, combining $m_{l+1}$ with $m_{l+2}$ and $m_{l+3}$ with $m_{l+4}$ (which increases storage requirements in $F$ ), and using as divisors the moduli $m_{l+1} m_{l+2}, m_{l+3} m_{l+4}, m_{l+5}, \cdots, m_{s}$, the average number of divisions that have to be performed approaches $11 / 8$ asymptotically from below. Thus, the effect of combining moduli in $F$ is to lower the critical value of $\tau$. Such a procedure would therefore be used when the available hardware in $V$ is smaller than required for keeping up with the speed of $F$ implied by an average of two divisions per solution candidate.

The high speeds which can be achieved by our method suggest its applicability for conversion of numbers from the modular number representation [7] to the conventional polyadic representation. Since this problem is of the Chinese remainder type, it seems possible to include in the sieve special solution hunting properties.

8. Conclusion. A method has been presented to sift numbers satisfying a set of linear congruences from among a large set of numbers. The important properties of the resulting special-purpose device are that a relatively large set of numbers is processed essentially within the time required for performing a shift of one position in an ordinary shift-register, and that no memory references are necessary. This leads to an overall speed gain of about three orders of magnitude over modern 
present-day computers such as the IBM 7090. By combining the device with a general-purpose computer, the size of problems that can be run is greatly increased with almost no decrease in speed.

\section{Appendix}

The Division Subroutine. For the purposes of this subroutine, written for the IBM 7090, we restrict the size of $N$ in (2) to a number representable by 72 binary bits.

The first 36 of these are called $\mathrm{HW}$, and the last 36 are called $L W$. The core of the subroutine consists of the following sequence, where it is assumed that the accumulator is cleared at the beginning. Every bit of the periodic patterns $e_{i}$ is stored in a separate word, denoted by $W M$, and the corresponding period is stored in $M$.

$\begin{array}{lll}L D Q & \text { (Load the } M Q) & H W \\ D V P & \text { (Divide) } & M \\ L D Q & \text { (Load the } M Q) & L W \\ D V P & \text { (Divide) } & M \\ P A C & \text { (Place complement of address } & 0,4 \\ & \text { in index register) } & \\ C L A & \text { (Clear add) } & W M, 4 \\ T M I & \text { (Transfer on minus) } & O U T\end{array}$

This sequence requires 36 cycles. For a quadratic sieve, the sequence has to be performed twice on the average for each solution candidate. Another 20 cycles are required for performing the multiplication and addition implied by (14) and bookkeeping. One cycle takes $2.18 \mu$ sec. Thus the subroutine requires about 200 $\mu$ sec.

Transfers from $V$ to $F$. A preliminary study of the $(F+V)$ organization based on the IBM 7090 as $F$ indicates that transfers from $V$ to $F$ can be effected in the manner of a data channel. Such a channel has a "Channel Address Counter" (CAC), from which addresses are transferred to the "Memory Address Register."

Suppose that the memory region bounded by addresses $K$ and $K+M$ is allocated for storing the value $n$ contained in $R$, and $L$ to $L+M$ for storing the contents of the $k$-register. We assume, for simplicity, that registers $R$ and $k$ do not exceed 36 bits. In its normal form, the $C A C$ contains an address of the form $K+i(0 \leqq i \leqq M)$. Three flip-flops $F F 1, F F 2, F F 3$ are contained in $S C$. $F F 1$ records whether $F$ or $V$ was the last user of the buffer region of the memory. $F F 2$ and $F F 3$ define "full" and "empty" conditions of the buffer.

We adopt the following operating rules.

1. When $V$ wants to store into the memory, the $C A C$ is advanced by 1 if $F F 1=1$, and remains unchanged if $F F 1=0$. Then $n$ is stored at the address currently held in $C A C$, say $K+i$. Next $C A C$ is changed to $L+i$, and the contents of the $k$-register are stored. Then $C A C$ is set back to $K+i$. After execution of these stores, $F F 1$ is set to 1 .

2. When $F$ wants to fetch a pair of new values from the memory, the $C A C$ is decreased by 1 if $F F 1=0$, and is left unchanged if $F F 1=1$. The address (con- 
tents of $C A C$ ) is forced into the $F$ Memory Address Register as a consequence of recognition of a special instruction in $F$ by the Supervisory Control. Both the value $n$ and the corresponding contents of the $k$-register are then fetched by the previously described $K-L$ interchange, and $C A C$ is set back to $K+i$. At the end of the fetching operations, $F F 1$ is set to 0 .

Thus $F$ always handles first the latest information brought in from $V$. If at any time $C A C$ holds the address $K+M$, and if $F F 1=1$, then $F F 2$ is set, which prevents $V$ from storing into the memory. (Of course for sufficiently large $l$, such an occurrence is very rare.) $F F 2$ is reset by the resetting signal of $F F 1$. If at any time $C A C$ holds the address $K$, and if $F F 1=0$, then $F F 3$ is set, which prevents $F$ from fetching. $F F 3$ is reset by the setting signal of $F F 1$.

Preliminary studies of this mode of transfer indicate that transfer of the first word takes at most two cycles, and the second takes one cycle. Thus the transfer of $n$ and the contents of the $k$-register from $V$ to $F$ requires approximately $7 \mu$ sec.

Department of Mathematics

Princeton University

Princeton, New Jersey

Department of Engineering

University of California

Los Angeles 24, California

Department of Mathematics

University of Oregon

Eugene, Oregon

Department of Engineering

University of California

Los Angeles 24, California

1. D. H. Lehmer, "A photo-electric number sieve," Amer. Math. Monthly, v. 40, 1933, p. $401-406$

2. D. H. LEHMER, "A machine for combining sets of linear congruences," Math. Ann. v. 109,1934, p. 661-667. p. 6-14

3. D. H. LEHMER, "The sieve problem for all-purpose computers," MTAC, v. 7,1953 ,

4. D. H. LEHMER, “Teaching combinatorial tricks to a computer," Proceedings of Symposia in Applied Mathematics, v. X, American Mathematical Sociẹty, Providence, R.I., $1960, p$. 179-193.

5. D. H. Lehmer, private communication, June 1960.

6. G. Estrin, "Organization of computer systems-the fixed plus variable structure computer," Proceedings of the Western Joint Computer Conference, May 1960, p. 33-37.

7. A. Svoboda, "Rational numerical system of residual classes," Stroje $\mathrm{Na} Z$ pracovani Informaci. (Czechoslovakia), v. 5, 1957, p. 9-47. 